\title{
Importancia y estado actual de los registros de cáncer de base poblacional en Perú
}

\author{
Importance and current status of population based cancer registries in Peru
}

\section{Sr. Editor:}

El cáncer es un problema de salud pública significativo dada la gran carga de enfermedad y mortalidad que produce (1). Para el año 2018, GLOBOCAN estimó 18,1 millones de casos nuevos y 9,6 millones de muertes por cáncer; además, se calcula que la prevalencia en los últimos 5 años alcanza los 15 millones de personas. En Perú, de acuerdo a estimados de la OMS para el 2015, cáncer fue la primera causa de muerte en personas menores de 70 años $(1,2)$.

En este contexto, los registros de cáncer son herramientas fundamentales en el control de esta patología. Existen tres tipos de registros de cáncer: hospitalarios, de patología y de base poblacional.

Los registros de base hospitalaria realizan la recolección de información de todos los casos de cáncer tratados en uno o más hospitales; estos datos son útiles para analizar el desempeño clínico y administrativo; asimismo, permite evaluar estándares de calidad y comparación entre centros hospitalarios.

Los registros de patología se basan en la recolección de datos provenientes de laboratorios de histopatología, proporcionando una impresión rápida del comportamiento del cáncer; sin embargo, representan una muestra sesgada de la población ya que no representan la totalidad de pacientes, por ejemplo, los que cuentan con diagnósticos radiológicos, de laboratorio, u otros (3).

Por otra lado, los registros de cáncer de base poblacional (RCBP) permiten la recolección sistemática de la información sobre todas las neoplasias malignas que se presentan en una población geográficamente definida y es el principal suministro de información sobre la tasa de incidencia de cáncer en el área de estudio; los datos provienen de todas las instituciones prestadoras de salud (públicas y privadas), por lo tanto, estos pueden proporcionar un insumo esencial para evaluar la carga de enfermedad en la población. Adicionalmente, la información recolectada es útil para determinar la etiología, factores de riesgo, investigación, planificación en salud, así como el impacto de las decisiones en el manejo del cáncer (3).

La creación de los RCBP nace del reconocimiento de la necesidad y conveniencia de contar con uno por parte de la población y sus autoridades. Planificar y desarrollar estos registros requiere de la participación de los principales actores del sector salud cuyo trabajo coordinado es esencial para garantizar el acceso a la información, sostenibilidad y éxito del registro. El liderazgo, contar con un equipo multidisciplinario motivado, el presupuesto suficiente, logística, infraestructura, así como la selección de un ámbito poblacional determinado, son claves para el desarrollo de los RCBP. Asimismo, la Agencia Internacional de Investigación en Cáncer (IARC, por sus siglas en inglés) ha planteado criterios de calidad en RCBP los cuales incluyen: comparabilidad, integridad, validez y puntualidad $(2,3)$.

El Perú es un país que posee RCBP establecidos, existen registros en Lima, Trujillo y Arequipa los cuales, asociados a contribuciones regionales, cubren el $36 \%$ de la población $(1,4)$. El RCBP de Lima Metropolitana con sede en el Instituto Nacional de Enfermedades Neoplásicas inició sus actividades en el año 1968, actualmente cuenta con asesoría técnica de IARC y su inclusión en el volumen XI de Cancer Incidence in Five Continents, así como GLOBOCAN 2018 refleja la calidad de los datos publicados, la última versión publicada en el 2016 recoge los datos del periodo 2010-2012 (5).

El RCBP de Arequipa fue fundado en el año $2001 \mathrm{e}$ inició sus actividades el 2002, a la fecha cuenta con 3 publicaciones: "Reporte de Cáncer Hospitalario 2000- 
2001", "Registro de cáncer poblacional de Arequipa 2002 - 2003" y "Registro de Cáncer Poblacional de Arequipa 2004-2007" (6). Por su parte, el RCBP de Trujillo inició como registro histológico en 1983, un año después inició sus labores como RCBP hasta 1990, año en el que fue reconocido por el Ministerio de Salud, tuvo como sede los principales hospitales de la ciudad. Sin embargo, la última publicación oficial del RCBP de Trujillo evaluó el periodo 1996-2002, dejando una amplia brecha hasta la actualidad $(4,7)$.

Recientemente, se creó el Comité de Registro de Base Poblacional de Trujillo Metropolitano que preside el Instituto Regional de Enfermedades Neoplásicas con sede en Trujillo, dicha iniciativa pretende reactivar el RCBP de Trujillo (gráficos 1 y 2).

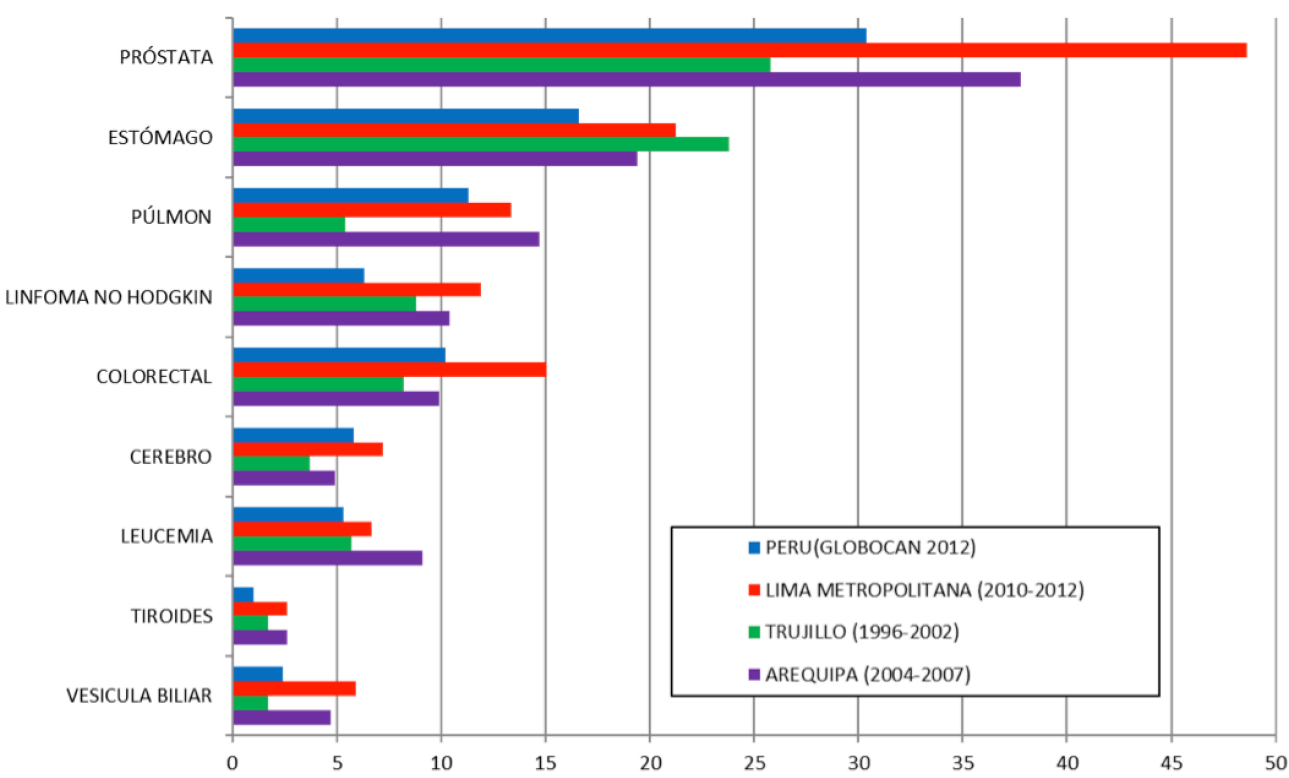

Gráfico 1. Tasa de incidencia estandarizada para varones por 100000 habitantes. Comparación entre los RCBP de Lima Metropolitana, Trujillo, Arequipa y estimados de Globocan IARC.

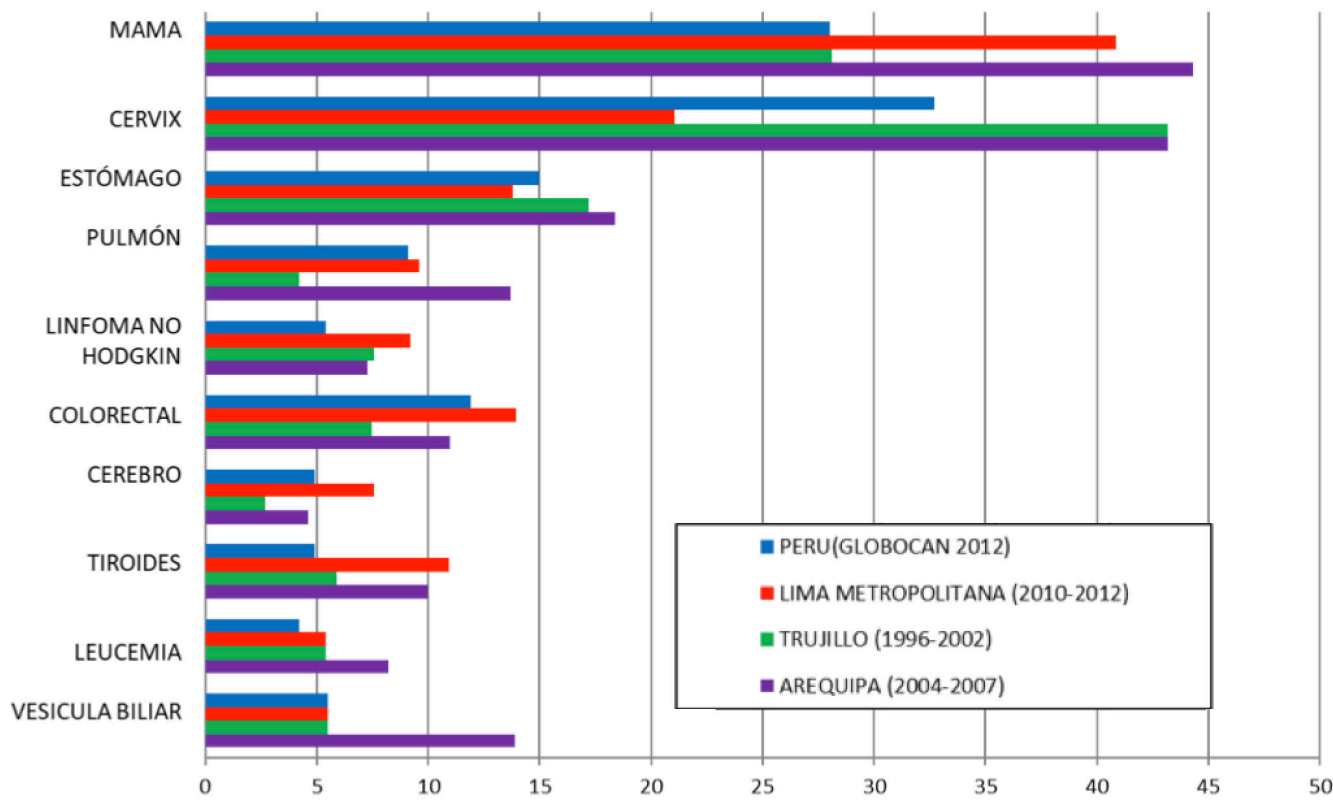

Gráfico 2. Tasa de incidencia estandarizada para mujeres por 100000 habitantes. Comparación entre los RCBP de Lima Metropolitana, Trujillo, Arequipa y estimados de Globocan IARC. 
Actualmente el Perú se encuentra en una transición demográfica la cual tiene un gran impacto en la incidencia y prevalencia del cáncer (4); en tal sentido, los RCBP son elementos primordiales para plantear un programa de control de cáncer acorde a nuestra realidad. Se debe reconocer el valor de los RCBP como herramientas útiles y necesarias para entender el comportamiento del cáncer en nuestra población. Asimismo, la información recolectada, al ser de buena calidad, nos permitirá plantear mejores políticas de salud y evaluar su impacto en beneficio de los pacientes con enfermedades neoplásicas en nuestro país.

\section{Jorge Luna-Abanto 1,a, Eduardo Payet 2,b,c}

\section{Correspondencia:}

Jorge Luna-Abanto

Avenida Angamos Este 2520 Surquillo. Lima, Perú.

Teléfono: 51982366213

Correo electrónico: Jorgelunaabanto@gmail.com

\section{REFERENCIAS BIBLIOGRÁFICAS}

1. Sarria-Bardales G, Limache-García A. Cancer control in Peru: a comprehensive approach to a public health issue. Rev Peru Med Exp Salud Publica. 2013; 30(1):93-8.
2. Bray F, Ferlay J, Soerjomataram I, Siegel RL, Torre LA, Jemal A. Global Cancer Statistics 2018: GLOBOCAN Estimates of Incidence and Mortality Worldwide for 36 Cancers in 185 Countries. CA Cancer J Clin. 2018; 68(6):394-424. doi: 10.3322/ caac. 21492

3. Bray F, Znaor A, Cueva P, et al. Planificación y desarrollo de registros de cáncer de base poblacional en los países de ingresos bajos y medios. Lyon: International Agency for Research on Cancer; 2015.

4. Piñeros M, Ramos W, Antoni S, et al. Cancer patterns, trends, and transitions in Peru: a regional perspective. Lancet Oncol. 2017; 18(10):e573-e586.

5. Payet E, Pérez P, Poquioma E, Díaz E. Registro de Cáncer de Lima Metropolitana. Incidencia y Mortalidad 2010 - 2012. Lima: Instituto Nacional de Enfermedades Neoplásicas; 2016.

6. Medina LE, Rodríguez B. Registro de Cáncer Poblacional de Arequipa 2004-2007. Arequipa: Instituto Regional de Enfermedades Neoplásicas del Sur; 2011.

7. Albújar P. El cáncer en Trujillo, 1996-2002: incidencia y mortalidad. Registro de Cáncer de Base Poblacional de Trujillo, Perú. Trujillo: Gerencia Regional de Salud de La Libertad; 2006.

Recibido: 11/02/2019

Instituto Nacional de Enfermedades Neoplásicas. Lima, Perú.

Comisión del Registro de cáncer de Lima Metropolitana. Lima, Perú.

Médico Residente de Cirugía Oncológica;

Presidente;

Doctor en Medicina. 